\title{
Identification of Microscopic Damage Law of Rocks Through Digital Image Processing of Computed Tomography Images
}

\author{
Zhe Qin $^{1,2}$, Yu Zhang ${ }^{1,2}$, Sheng Zhang ${ }^{1,2}$, Jingwei Zhao ${ }^{1,2 *}$, Tengfei Wang ${ }^{1,2}$, Ke Shen ${ }^{3}$ \\ ${ }^{1}$ College of Civil Engineering and Architecture, Shandong University of Science and Technology, Qingdao 266590, China \\ ${ }^{2}$ Shandong Key Laboratory of Civil Engineering Disaster Prevention and Mitigation, Shandong University of Science and \\ Technology, Qingdao 266590, China \\ ${ }^{3}$ Daqin Railway Co., LTD., Taiyuan Public Works Section, Taiyuan 030013, China
}

Corresponding Author Email: skd991560@sdust.edu.cn

https://doi.org/10.18280/ts.360407

Received: 2 March 2019

Accepted: 5 July 2019

\section{Keywords: \\ digital image processing (DIP), geotechnical engineering, computed tomography $(C T)$ scanning, representative elementary volume $(R E V)$, microscopic damages}

\begin{abstract}
This paper introduces digital image processing (DIP) to geotechnical field, aiming to disclose the microscopic damage law of rocks under cyclic water invasion. Firstly, the altered granite specimens under cyclic water invasion were subjected to computed tomography (CT) scanning, producing cross-sectional images. These images then underwent noise removal and threshold segmentation. The pores and cores, rock foundation, and high-density nodules were identified accurately in the processed images, reflecting the microstructure of the original rock mass. Based on the processed images, the 3D rock cores were reconstructed, and a $200 \times 200 \times 200$ representative elementary volume $(\mathrm{REV})$ was extracted from each rock core. The analysis results show that, with the growing number of water invasion cycles, the surface porosity and non-closed surface porosity continued to increase, while the closed surface porosity first increased and then declined. This research lays a theoretical basis for applying the DIP in geotechnical field.
\end{abstract}

\section{INTRODUCTION}

Digital image processing (DIP) is the use of computer algorithms to enhance, segment, restore, encode and compress images. This emerging technology has been widely applied in such field as biomedicine, communication and industrial engineering, yielding marked results in these engineering fields [1-4]. For example, many DIP methods have been adopted to study material properties $[5,6]$, ranging from scanning electron microscope (SEM) to computed tomography (CT).

DIP has become a research hotspot since its birth. On basic image processing, Gupta et al. [7] compared discrete wavelets (e.g. the Haar wavelet and the Daubechies wavelet) in the compression of static images, and evaluated the image transform effects by mean squared error (MSE) and energy retention (ER), revealing that the Daubechies wavelet outperforms the other discrete wavelets in ER.

Many scholars have relied on the DIP to detect defects of materials. For example, Chrysafi et al. [8] used active infrared thermography (IRT) to detect the defects of carbon fiber reinforced plastics (CFRPs), conducted mathematical processing and wavelet transform of the 2D thermograms, and found that 1D Fourier transform and 1D wavelet transform are the best methods to capture cracking effect. Rodríguez-Martín et al. [9] detected cracks in steel welding with a thermographer, proposed a depth prediction method for welding-induced surface cracks, and analyzed the correlation between infrared data and crack propagation data based on 3D megaphotographs. Sfarra et al. [10] inspected impacted laminates by different nondestructive testing (NDT) methods, such as infrared thermography (IRT), near-infrared reflectography (NIRR) and near-infrared transmittography (NIRT), accelerated the aging of impacted laminates in an environmental chamber, and inspected them again by the IRT and digital speckle photography (DSP), revealing the structural changes on these laminates after accelerated aging.

In addition, DIP methods are essential to detecting and identifying microscopic damage in geotechnical engineering. The DIP can acquire images on the microstructure changes of rocks in a nondestructive manner, providing comprehensive microscopic information (e.g. composition, pore development, and pore structure) of the rocks from multiple angles. Through 3D digital image correlation (DIC), Munoz et al. [11] conducted quasi-static monotonic uniaxial compression tests on Hawkesbury sandstone specimens, used digital cameras to capture specimen deformation, and thus determine the strain field and strain localization pattern. Chen et al. [12, 13] generated high-resolution SEM images of altered granite specimens, examined the internal structure and composition of the specimens, and discussed the microscopic damage law of altered granite.

However, the application of DIP in geotechnical engineering is far from mature. The exiting studies are often based on 2D images or low-resolution 3D images of rock specimens. There is not yet a realistic illustration of the microscopic damages of rocks. To disclose the exact microscopic damage law of rocks, this paper obtained highresolution cross-sectional images of altered granite under cyclic water invasion, using a high-precision CT scanner. The images were subjected to noise removal and threshold segmentation, and then adopted for 3D image reconstruction. After extracting the representative elementary volume (REV), the author investigated the development of microscopic pores 
and cracks of the rock, and identified the 3D damage law of the microstructure of the rock. With the aid of CT scanning, our research reveals how cyclic water invasion damages the microstructure of rocks. The research findings pave the way for further research on megascopic mechanical properties, and lay the theoretical basis for microscopic study of geotechnical engineering.

\section{CYCLIC WATER INVASION TESTS AND CT SCANNING}

\subsection{Cyclic water invasion tests}

Under long-term cyclic water invasion, altered granite will witness changes in its mechanical properties, porosity, etc. This paper attempts to simulate the cyclic water invasion of altered granite in lab, and reveal the microstructure changes of the rock under different water invasion cycles.

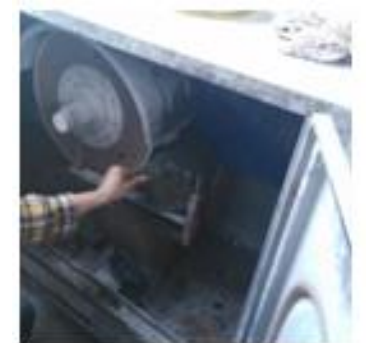

(a) Cutting

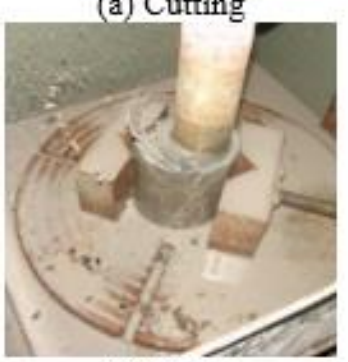

(c) Coring

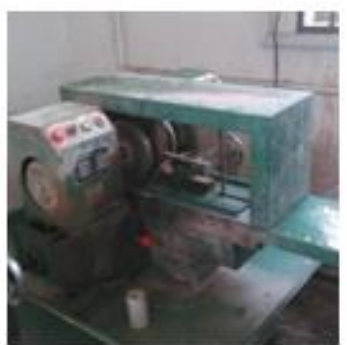

(b) End-face leveling

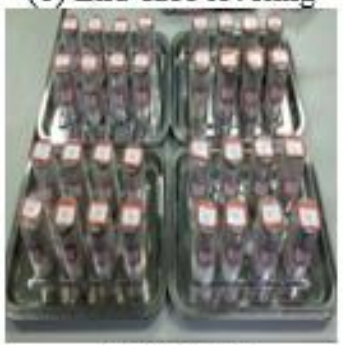

(d) Sorting
Figure 1. Preparation of rock specimens

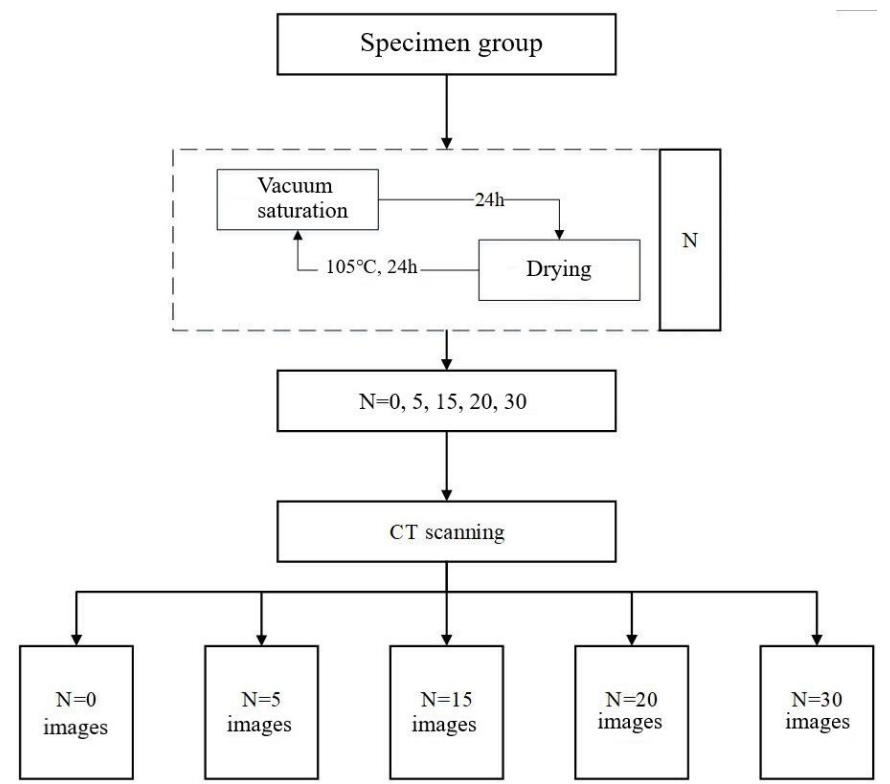

Figure 2. Cyclic water invasion tests (N: the number of water invasion cycles)
For this purpose, 12 standard rock specimens $(100 \mathrm{~mm} \times \varphi 50 \mathrm{~mm})$ were prepared, and divided into 6 groups. Before water invasion tests, all rock specimens were saturated with water in the vacuum. The first five groups were subjected to water invasion for $0,5,15,20$ and 30 cycles, and numbered as Y0, Y5, Y15, Y20 and Y30, respectively. The remaining group was taken as the control group.

Each cycle of water invasion was implemented in two steps: the rock specimens after vacuum saturation were soaked for $24 \mathrm{~h}$ in an immersion chamber at room temperature, and the soaked specimens were relocated to a drying oven to be dried for $24 \mathrm{~h}$ at $105{ }^{\circ} \mathrm{C}$.

After completing water invasion tests, the rock specimens were subjected to CT scanning to obtain their cross-sectional images. The procedure of specimen preparation and water invasion tests is explained in Figures 1 and 2 above.

\subsection{CT scanning}

After each cyclic water invasion test, the rock specimens were placed into a multiscale CT core scanner (Figure 3 ) for CT scanning. Before the scanning, the parameters of the scanner were fully calibrated.

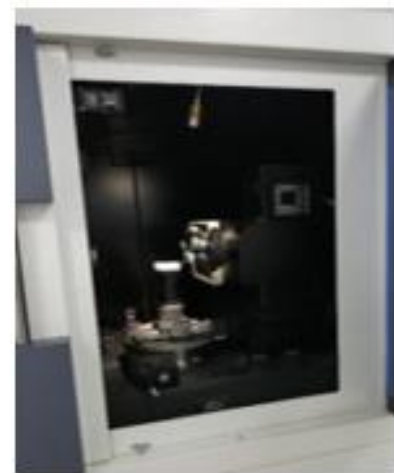

(a) Specimen placement

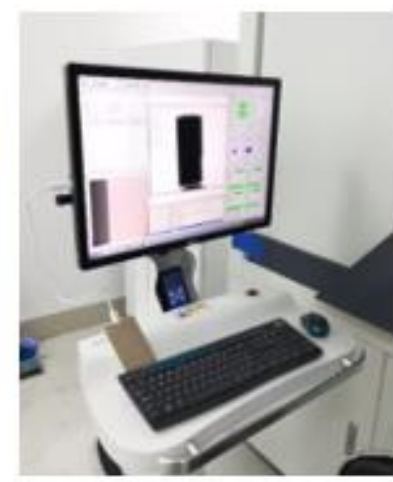

(b) CT scanning
Figure 3. Photos of CT scanning

The parameter setting of the multiscale CT core scanner directly bears on the results of CT scanning. Unreasonable parameters will slow down the scanning, waste resources and affect the quality of scan images, making it difficult to identify the microstructure inside the rock. Therefore, pre-scanning was performed with the scanner on the rock specimens to determine reasonable CT scan parameters, which can restore the micro-features of the specimens accurately and ensure the efficiency of the tests. Here, the scan spacing is set to $71.42 \mu \mathrm{m}$ and the image resolution is set to $79.71 \mu \mathrm{m}$ (pixel size). The CT scan images on the top, middle and bottom cross-sections of each specimen group are presented in Figures 4-8. For the lack of space, this paper only displays the cross-sectional images of only one specimen from each group.

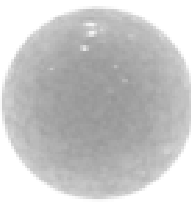

(a)

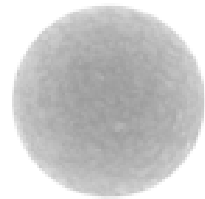

(b)

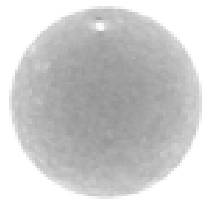

(c)
Figure 4. CT scan images of specimen YO 


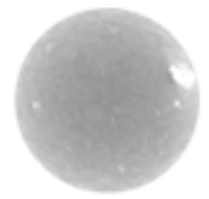

(a)

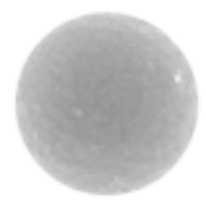

(b)

(c)

Figure 5. CT scan images of specimen Y5

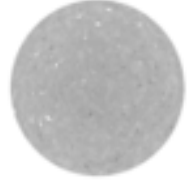

(a)

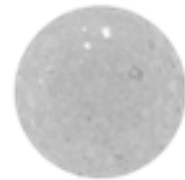

(b)

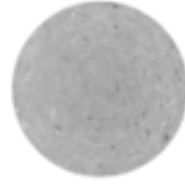

(c)
Figure 6. CT scan images of specimen Y15

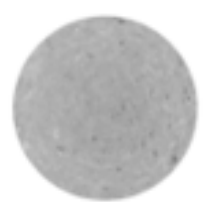

(a)

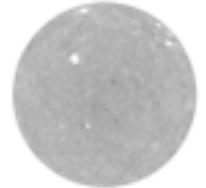

(b)

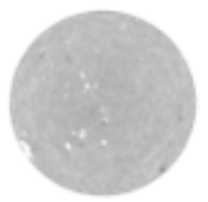

(c)
Figure 7. CT scan images of specimen Y20

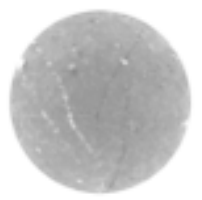

(a)

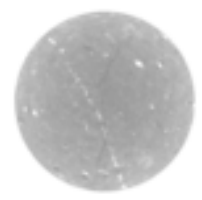

(b)

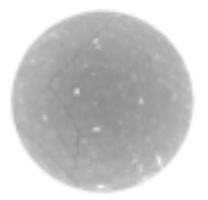

(c)
Figure 8. CT scan images of specimen Y30

\section{DIGITAL IMAGE PROCESSING}

\subsection{Noise removal}

Before 3D reconstruction of rock cores, the cross-sectional images of each specimen underwent a series of DIP processes to make them more realistic. These processes mainly include the filtering, binarization and threshold segmentation of grayscale images.

The filtering is necessary because the CT scan images often contain many noises, such as salt-and-pepper noise and other physical noises. The purpose of filtering is to reduce the noises produced by instruments and the surrounding environment, and improve the imaging quality, laying a good basis for subsequent image segmentation.

The common filtering techniques include smoothing, contrast enhancement and edge detection [14]. Considering the situation of our tests and cross-sectional images, three methods are suitable for filtering the cross-sectional images of our specimens: digital image filters, e.g. Gaussian filter, median filter and mean filter [15].

(1) Gaussian filter is a linear smoothing filter. The basic principle is to replace the gray value of each pixel in the $2 \mathrm{D}$ image with the weighted mean of the gray values of that pixel and its neighborhood, thus eliminating normally distributed noises.

(2) Median filter is a nonlinear smoothing technique that denoises images. Median filtering basically substitutes the gray value of each pixel in the 2D image with the median of gray values of nearby pixels, and removes isolated noisy points by approximating the actual value with nearby values.

(3) Mean filter is the simplest linear smoothing tool. In the course of mean filtering, a box is firstly set up around the target pixel to cover eight nearby pixels, and then the mean pixel value of the eight nearby pixels is used to replace the original value of the target pixel.

The filtered images of the three filters are compared in Figure 9. In this paper, the smoothing and contrast enhancement are adopted to filter the cross-sectional images of each specimen.

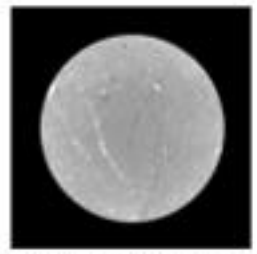

(a) Gaussian filter

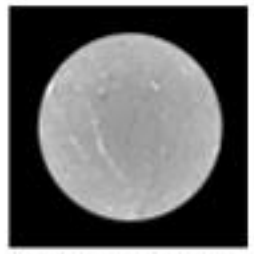

(b) Median filter

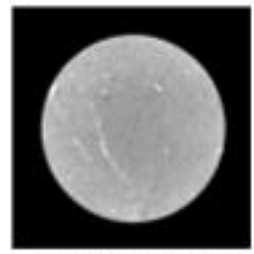

(c) Mean filter
Figure 9. Filtered images

\subsection{Threshold segmentation}

Pores and cracks are the main influencing factors of the megascopic mechanical properties, conductivity and permeability of rock $[16,17]$. Hence, it is very meaningful to identify the pores and cracks in the cross-sectional images, before exploring the microscopic damage law of altered granite. Rocks often contain various minerals and multiphase materials. In our research, the target rock, altered granite, is mainly made up of quartz, mica, feldspar, and high-density nodules. Geotechnically speaking, the mechanical strength of rocks is directly affected by structural bodies and planes, that is, the rock foundation and pores and cracks.

Considering the heavy presence of high-density mineral nodules in altered granite, the rock specimens were treated as the combination of rock foundation, pores and cracks and high-density nodules. The grayscale cross-sectional images were binarized based on pores and cracks (Figure 10), and high-density nodules. To binarize a grayscale image, a reasonable threshold should be determined to segment the image into multiple parts and to identify the different components in the image. The threshold is usually selected based on the grayscale image: the peak values in the image often appear in the optimal range of threshold segmentation.

In this paper, the pores and cores, and high-density nodules are segmented from the grayscale images by the watershed algorithm [18, 19] (Figure 11). In this algorithm, the gray value is considered as altitude, and each local minimum and its affected area are treated as a drainage basin. Different drainage basins are divided by watersheds. Each watershed represents the maximum gray values of the input image. To obtain edge information, gradient image is usually used as the input image:

$$
\begin{aligned}
& g(x, y)=\operatorname{grad}(f(x, y)) \\
& =\{[f(x, y)-f(x-1, y)] 2[f(x, y)-f(x, y-1)] 2\} 0.5
\end{aligned}
$$


where, $f(x, y)$ is the original image; $\operatorname{grad}\{\quad\}$ is the gradient operation.

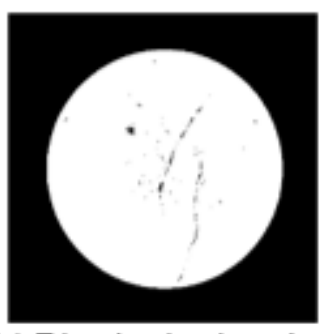

(a) Binarization based on pores and cracks

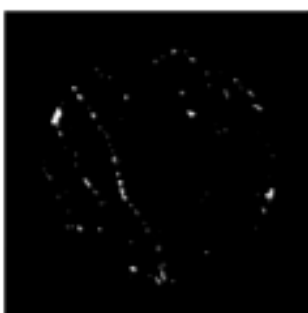

(b) Binarization based on high-density nodules

Figure 10. Binarization of grayscale images

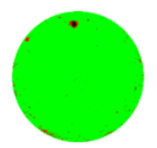

Y0

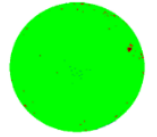

Y5

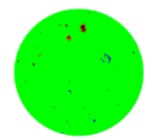

Y15

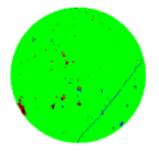

Y20

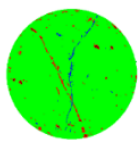

Y30

Figure 11. Results of threshold segmentation (Green: Rock foundation; Blue: Pores and cores; Red: High-density nodules)

\subsection{D reconstruction of rock cores}

After noise removal and threshold segmentation, the crosssectional images of each specimen were superimposed to reconstruct the $3 \mathrm{D}$ rock core. The $3 \mathrm{D}$ reconstruction was achieved with the aid of the other modules in the DIP software. The reconstruction is essentially a $3 \mathrm{D}$ rendering of the crosssectional images, which visually reproduces the rock core [20]. The reconstructed model helps to disclose the microscopic features of the rock under cyclic water invasion (Figure 12).

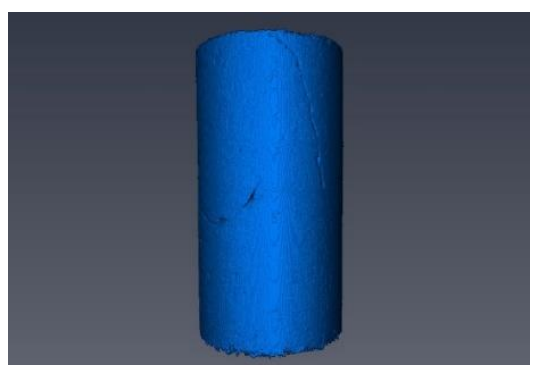

Figure 12. The reconstructed $3 \mathrm{D}$ model of specimen $\mathrm{Y} 30$

\section{SELECTION ELEMENTARY ANALYSIS}

OF VOLUMES

\subsection{The concept of the REV}

The REV of the reconstructed $3 \mathrm{D}$ model is critical to the understanding of the heterogeneous pore structure of the reservoir. The REV theory was proposed by Bear in 1972 based on the continuum hypothesis [21]. This theory exposes the limitations on the traditional practice of applying the continuum methods for porous media like rock mass.

Studies have shown that, the parameters of an object (e.g. modulus of deformation, permeability coefficient, coordination number and porosity) will fluctuate by different degrees, with the continued expansion of the regions of interest (ROIs) [22]. This trend fully reflects the anisotropy and non-uniformity of the rock mass (Area I, Figure 13). However, the parameters will no longer fluctuate, once the ROIs increased to a critical value (Area II, Figure 13). The volume in Area II can be called the REV. If the object is anisotropic, the parameters will fluctuate again with further expansion of the ROIs (Area III, Figure 13). The critical value between Areas I and II is the minimum REV ( $\left.\operatorname{REV}_{\min }\right)$.

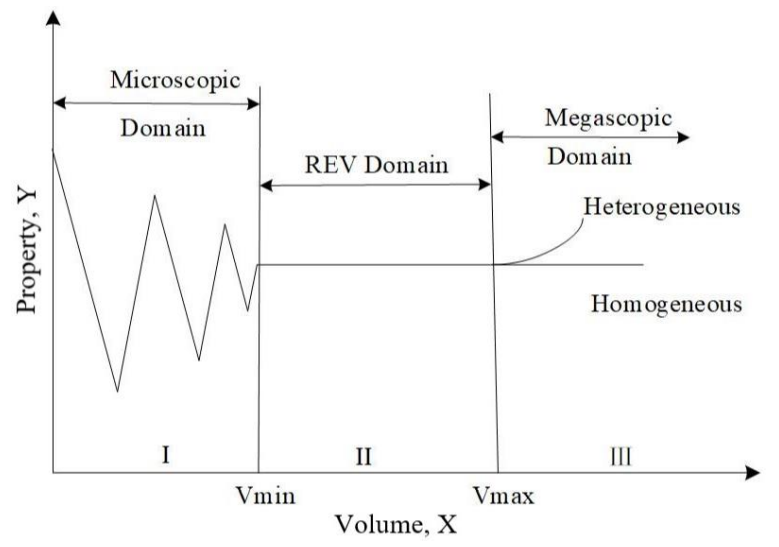

Figure 13. The definition of the REV

By its definition, the REV must be large enough to contain sufficient information about the global properties and small enough to remain in the microscale [23]. In essence, the REV is a measure of spatial uniformity, and reflects the unity of opposites like microscale and mega-scale, discreteness and continuum, as well as stochasticity and certainty. A properly selected REV helps to learn the spatial variation of object features. In general, the test results are meaningful only if the $\mathrm{REV}$ is on the same scale with the specimen size.

In our research, lots of computing power will be wasted to reconstruct and render the entire specimen based on the crosssectional images. The selection of REV size not only reflects the microstructure of the rock, but also saves the computing power, facilitating the finite-element analysis of the rock cores.

\subsection{Selection of REV size}

In the light of our tests and research contents, the REV size was selected in three steps based on porosity, a key physical parameter of the altered granite under cyclic water invasion.

Step 1. Select a relatively small voxel at the center of each cross-sectional image (size: $750 \times 750$ ).

Step 2. Cut out a cube centering on the voxel, such as to contain the reconstructed rock core, and compute the porosity of that cube.

Step 3. Expand the cube centering on the voxel by changing the voxel size to $100,150,200,250,300,350$ and 400 , in turn. Compute the porosity of each cube and draw the porosity-cube size curve. As shown in Figure 14, the curve became flat when the porosity was stabilized. The minimum cube was taken as the REV size for each specimen. 


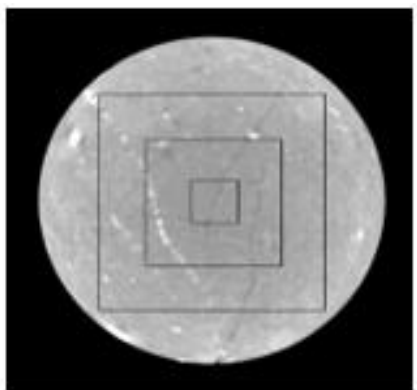

(a) Cropping of cross-sectional image

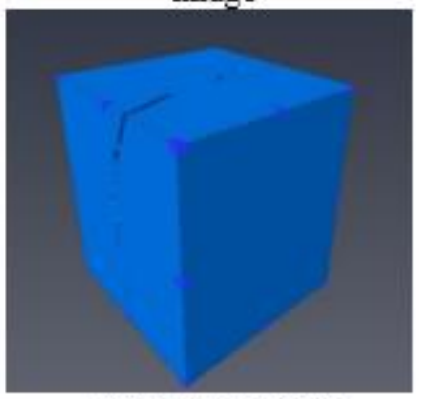

(d) 200-pixel cube

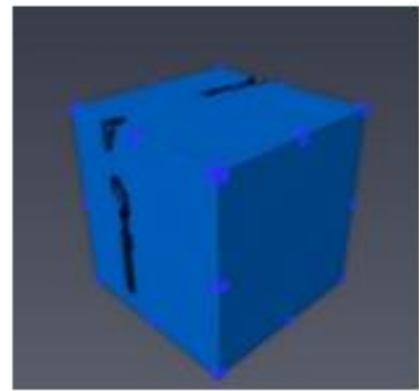

(b) 50-pixel cube

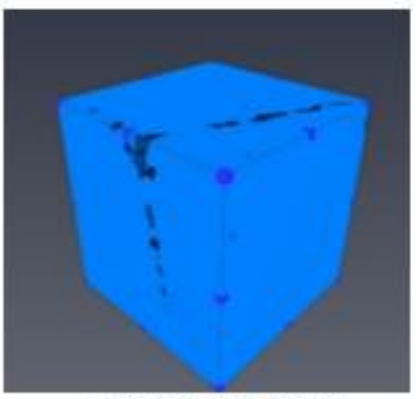

(e) 300-pixel cube

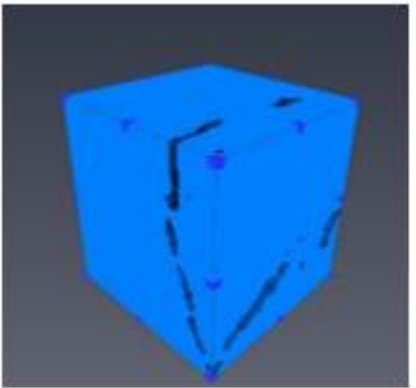

(c) 100-pixel cube

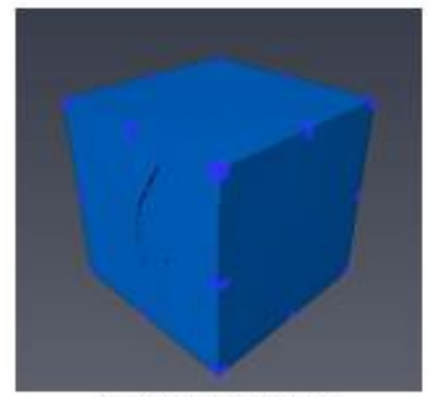

(f) 400-pixel cube

Figure 14. Selection of REV size

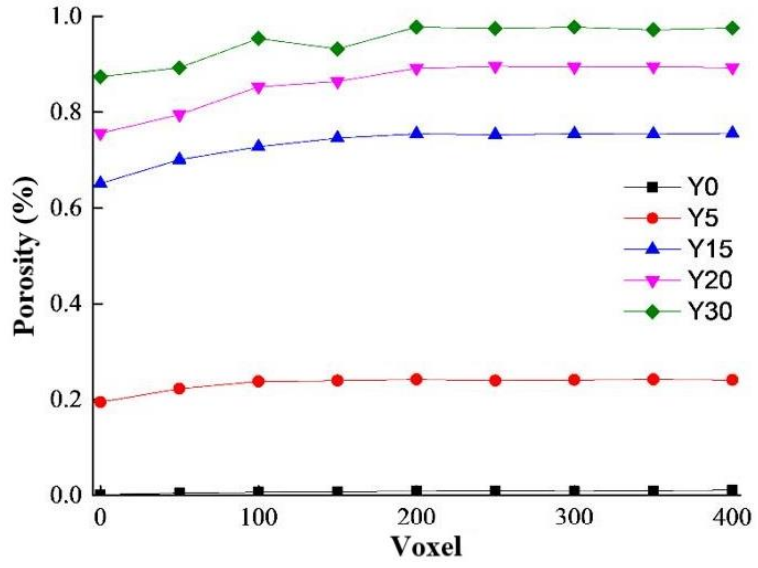

Figure 15. Relationship between porosity and voxel size

As shown in Figure 15, with the growth in voxel size, the porosities of all specimens exhibited a gradual increase. When the voxel size was 200 , the porosity of each specimen gradually stabilized and tended to fixed value. Hence, the REV size for specimens under different cycles of water invasion was determined as $200 \times 200 \times 200$.

\subsection{REV-based analysis of microscopic damage features}

The REV was extracted from the interior of each standard specimen to replace the specimen in further analysis (Figure 16). The replacement eliminates the accidental damages on specimen appearance in the tests, and greatly bolsters the efficiency of computing and analysis, saving a huge amount of computing resources.

Under cyclic water invasion, the rock damages are mainly manifested by the variation in pores and cracks. As a result, the REV cross-section was studied to determine the number, area, shape and distribution of pores and cracks, and to analyze the micro-damages induced by cyclic water invasion.

The pores and cracks of the rock are highly irregular. The REV of the same specimen may contain multiple heterogenous cross-sections. To solve the problem, a coordinate system was set up based on standard specimen (Figure 16), and the XOY cross-section of the REV was taken as the research object. Figures 17 21 provide the REV cross-sections at different number of water invasion cycles.

The comparison between Figures 17 21 shows that the specimen under zero water invasion enjoyed a dense structure, with almost no pore but a slight initial damage. With the growing number of water invasion cycles, more and more pores appeared, and the development and propagation of certain pores and cracks intensified the damages. At the $30^{\text {th }}$ cycle of water invasion, the internal cracks connected with each other, penetrating through the REV cross-section, and the cyclic water invasion caused marked damages to the specimen.

Judging by their morphology and distribution on the REV cross-section, the pores and cracks were either closed or not closed. The closed ones are mostly pores. Some of them are original pores in the rock, and some were induced by cyclic water invasion. The non-closed ones are mostly cracks. The cracks were entirely caused by cyclic water invasion. The two types of pores and cracks have similar yet slightly different causes. Both demonstrate the rock damages under cyclic water invasion. In the images of REV cross-sections, the damages are exhibited as the growing proportion of the area of pores and cracks. Hence, the surface porosity was defined to describe the damages.

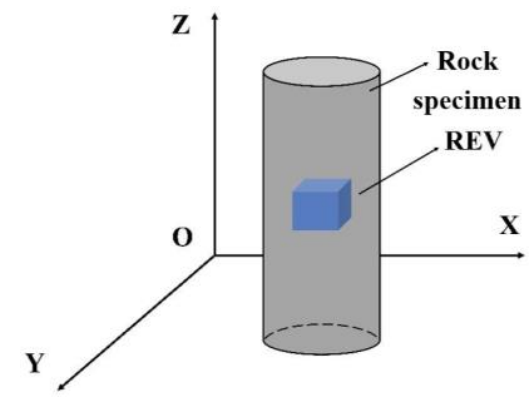

Figure 16. Specimen coordinate system 


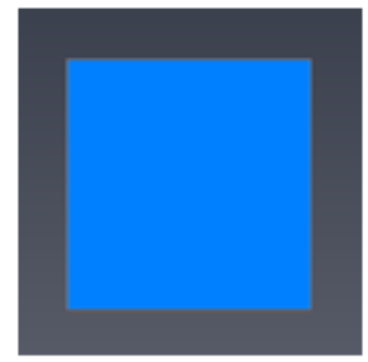

(a)

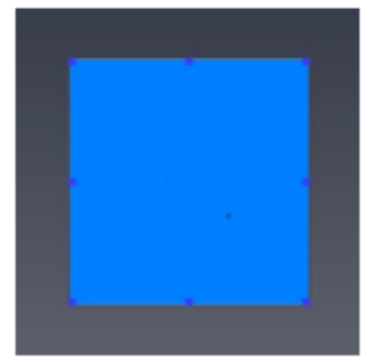

(c)

Figure 17. REV sections of specimen $Y 0$

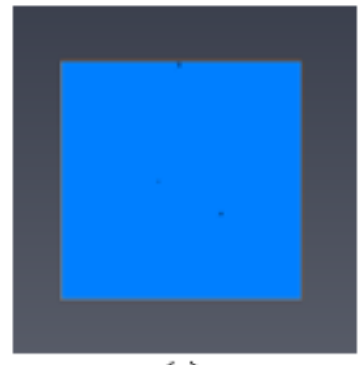

(a)

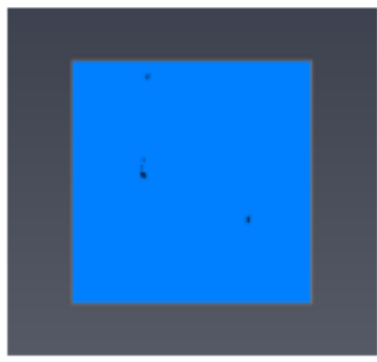

(b)

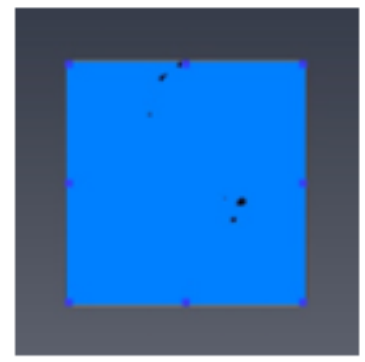

(c)

Figure 18. REV sections of specimen $\mathrm{Y} 5$

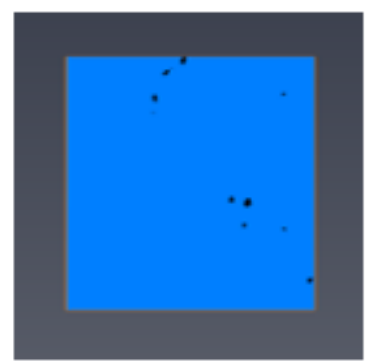

(a)

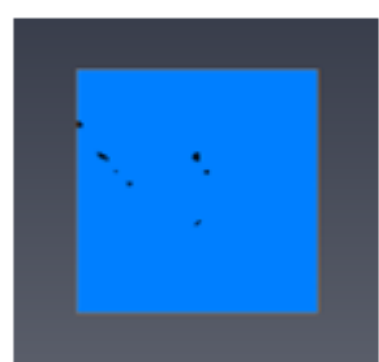

(b)

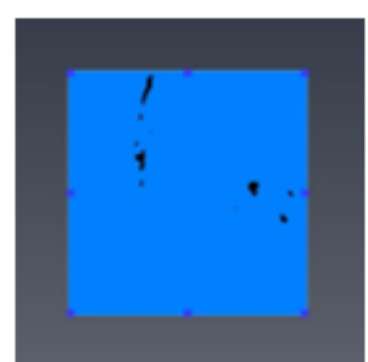

(c)

Figure 19. REV sections of specimen $Y 15$

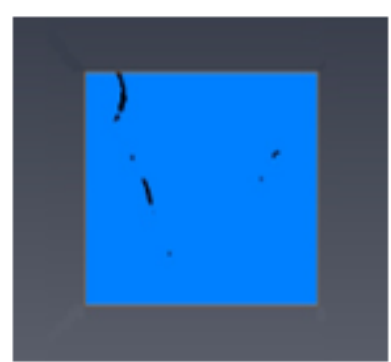

(a)

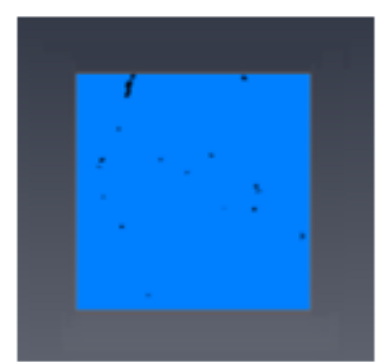

(b)

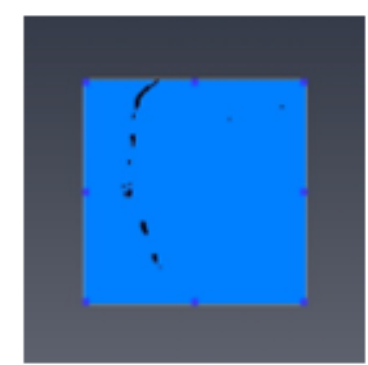

(c)

Figure 20. REV sections of specimen Y20

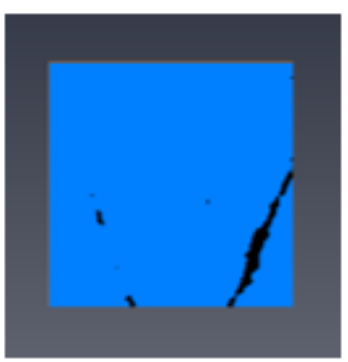

(a)

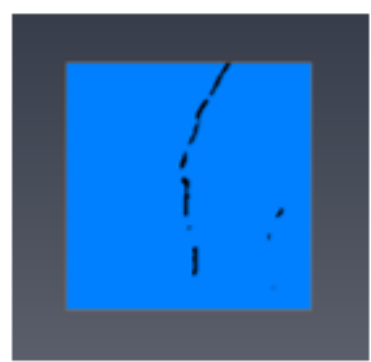

(b)

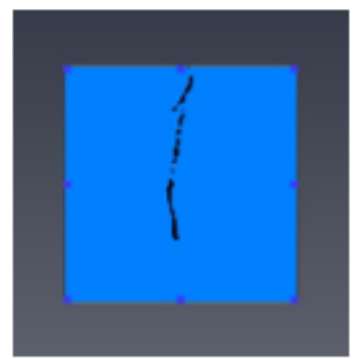

(c)

Figure 21. REV sections of specimen Y30 
Let $A, A_{r}$ and $A_{p}$ be the total area, rock foundation area, and pores and cracks area of a REV cross-section, respectively, where $A_{p}$ is the sum of the area of closed pores and cracks $A_{p_{1}}$ and that of non-closed pores and cracks. Based on the definition of porosity, the 2D surface porosity $r$ of rocks can be defined as:

$$
r=\frac{A_{p}}{A} \times 100 \%
$$

Thus, the closed surface porosity and non-closed surface porosity can be obtained by $r_{1}=\frac{A_{p_{1}}}{A} \times 100 \%$ and $r_{2}=\frac{A_{p_{2}}}{A} \times$ $100 \%$, respectively.

The closure of cores and pores may vary with the size of REV cross-section or change due to REV extraction. To solve the problem, the surface porosity, closed surface porosity and non-closed surface porosity were computed multiple times, and the mean values were taken as the final results. The REV (voxel) size of all rock cores was set to $200 \times 200 \times 200$, and 100 cross-sections were extracted from top to bottom by a certain interval. Each cross-section is $2.36 \mathrm{~cm}^{2}$ in size. The three surface porosities were computed for each cross-section. The mean results of each cross-section are displayed in Table 1 below.

Table 1. Results on surface porosities

\begin{tabular}{cccc}
\hline $\begin{array}{c}\text { Number of } \\
\text { water } \\
\text { invasion } \\
\text { cycles }\end{array}$ & $\begin{array}{c}\text { Closed } \\
\text { surface } \\
\text { porosity }(\%)\end{array}$ & $\begin{array}{c}\text { Non-closed } \\
\text { surface } \\
\text { porosity }(\%)\end{array}$ & $\begin{array}{c}\text { Surface } \\
\text { porosity } \\
(\%)\end{array}$ \\
\hline 0 & 0.010 & $<0.001$ & 0.010 \\
5 & 0.237 & 0.005 & 0.242 \\
15 & 0.613 & 0.137 & 0.755 \\
20 & 0.536 & 0.356 & 0.892 \\
30 & 0.315 & 0.663 & 0.978 \\
\hline
\end{tabular}

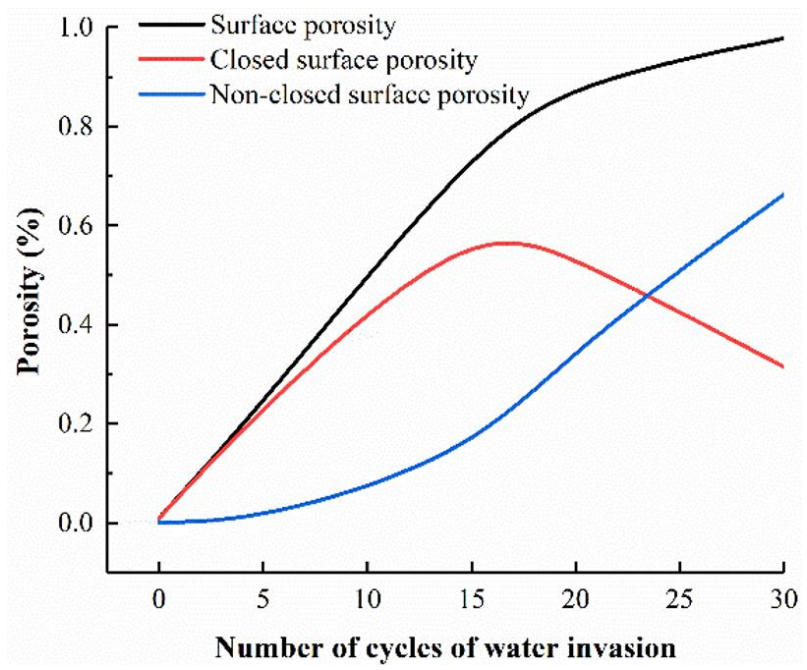

Figure 22. Relationship curves between number of water invasion cycles and surface porosities

As shown in Figure 22, the surface porosities of each REV increased with the number of water invasion cycles. On the state of pores and cracks, as the number of water invasion cycles increased from 0,5 to 15 , the closed surface porosity and non-closed surface porosity were both on the rise; as the number of water invasion cycles increased from 20 to 30 , the closed surface porosity decreased, while the non-closed surface porosity continued to grow. The main reason is that the rock damages induced by cyclic water invasion constantly increases, causing the internal pores to expand, connect and evolve into non-closed pores. Through the above analysis, the author had a preliminary understanding of the initiation, development and propagation of pores and cracks in the rock under cyclic water invasion, and quantified the microscopic damages caused by the cyclic water invasion.

\section{CONCLUSIONS}

This paper simulates the cyclic water invasion of altered granite in the lab, conducted CT scanning of the rock specimens with different number of water invasion cycles, and obtained the cross-sectional images of each specimen. Next, the microscopic features of the cross-sectional images were quantified and qualified through DIP. Finally, the 3D rock cores were reconstructed based on the cross-sectional images, and used to disclose the microscopic damage law of the rock cores. The main research results are as follows:

(1) The cross-sectional images were subjected to noise removal and threshold segmentation. On the one hand, the images were filtered by smoothing and contrast enhancement. On the other hand, a suitable threshold was determined based on binarized images, and used to distinguish between the rock foundation, pores and cracks, and high-density nodules.

(2) The processed cross-sectional images were adopted to reconstruct the $3 \mathrm{D}$ rock cores. The reconstructed cores are close to the original rock. In addition, the REV of the cores was set to $200 \times 200 \times 200$ with porosity as the judgment criterion.

(3) The microscopic damages of rock specimens were quantified according to the surface porosity, closed surface porosity and non-closed surface porosity of the REV. The results show that, with the growing number of water invasion cycles, the surface porosity and non-closed surface porosity continued to increase, while the closed surface porosity first increased and then declined.

\section{ACKNOWLEDGMENT}

This study is supported by the National Natural Science Foundation of China (No.51909149), Opening Foundation of Shandong Key Laboratory of Civil Engineering Disaster Prevention and Mitigation (CDPM2019ZR10), Scientific Research Foundation of Shandong University of Science and Technology for Recruited Talents (2017RCJJ050).

\section{REFERENCES}

[1] Han, Y., Hu, D., Matzar, L. (2014). Numerical computation of elastic properties for porous rocks based on CT-scanned images using direct mapping method. Journal of Petroleum Science and Engineering, 122: 346353. https://dx.doi.org/10.1016/j.petrol.2014.07.029

[2] Yu, Q., Yang, S., Ranjith, P.G., Zhu, W., Yang, T. (2016) Numerical modeling of jointed rock under compressive loading using X-ray computerized tomography. Rock Mechanics and Rock Engineering, 49(3): 877-891. https://dx.doi.org/10.1007/s00603-015-0800-4 
[3] Wei, M.D., Dai, F., Xu, N. W., Xu, Y., Xia, K. (2015). Three-dimensional numerical evaluation of the progressive fracture mechanism of cracked chevron notched semi-circular bend rock specimens. Engineering Fracture Mechanics, 134: 286-303. https://dx.doi.org/10.1016/j.engfracmech.2014.11.012

[4] Vidhyavathi, R.M. (2017). Principal component analysis (PCA) in medical image processing using digital imaging and communications in medicine (DICOM) medical images. International Journal of Pharma and Bio Sciences, $\quad 8(2)$ : 598-606. https://dx.doi.org/10.22376/ijpbs.2017.8.2.b.598-606

[5] Wang, J., Li, S.C., Li, L.P., Lin, P., Xu, Z.H., Gao, C.L. (2019). Attribute recognition model for risk assessment of water inrush. Bulletin of Engineering Geology and the Environment, $78(2)$ : 1057-1071. https://dx.doi.org/10.1007/s10064-017-1159-4

[6] Wang, G., Wang, K., Wang, S., Elsworth, D., Jiang, Y. (2018). An improved permeability evolution model and its application in fractured sorbing media. Journal of Natural Gas Science and Engineering, 56: 222-232. https://dx.doi.org/10.1016/j.jngse.2018.05.038

[7] Gupta, D., Choubey, S. (2015). Discrete wavelet transform for image processing. International Journal of Emerging Technology and Advanced Engineering, 4(3): 598-602.

[8] Chrysafi, A.P., Athanasopoulos, N., Siakavellas, N.J. (2017). Damage detection on composite materials with active thermography and digital image processing. International Journal of Thermal Sciences, 116: 242-253. https://dx.doi.org/10.1016/j.ijthermalsci.2017.02.017

[9] Rodríguez-Martín, M., Lagüela, S., González-Aguilera, D., Martinez, J. (2015). Prediction of depth model for cracks in steel using infrared thermography. Infrared Physics \& Technology, 71: 492-500. https://dx.doi.org/10.1016/j.infrared.2015.06.013

[10] Sfarra, S., Regi, M., Santulli, C., Sarasini, F., Tirillò, J., Perilli, S. (2016). An innovative nondestructive perspective for the prediction of the effect of environmental aging on impacted composite materials. International Journal of Engineering Science, 102: 55-76. https://dx.doi.org/10.1016/j.ijengsci.2016.02.009

[11] Munoz, H., Taheri, A., Chanda, E.K. (2016). Pre-peak and post-peak rock strain characteristics during uniaxial compression by 3D digital image correlation. Rock Mechanics and Rock Engineering, 49(7): 2541-2554. https://dx.doi.org/10.1007/s00603-016-0935-y

[12] Chen, X., He, P., Qin, Z. (2018). Damage to the microstructure and strength of altered granite under wetdry cycles. Symmetry, 10(12): 716. https://dx.doi.org/10.3390/sym10120716

[13] Qin, Z., Fu, H., Chen, X. (2019). A study on altered granite meso-damage mechanisms due to water invasion- water loss cycles. Environmental Earth Sciences, 78(14): 428. https://dx.doi.org/10.1007/s12665-019-8426-6

[14] Regalia, P. (2018). Adaptive IIR filtering in signal processing and control. Routledge. https://dx.doi.org/10.1201/9781315136653

[15] Gavili, A., Zhang, X.P. (2017). On the shift operator, graph frequency, and optimal filtering in graph signal processing. IEEE Transactions on Signal Processing, 65(23): 6303-6318. https://dx.doi.org/10.1109/TSP.2017.2752689

[16] Bidgoli, M.N., Jing, L. (2014). Water pressure effects on strength and deformability of fractured rocks under low confining pressures. Rock Mechanics and Rock Engineering, 48(3): 971-985. https://dx.doi.org/10.1007/s00603-014-0628-3

[17] Wu, Y., Tahmasebi, P., Lin, C., Munawar, M.J., Cnudde, V. (2019). Effects of micropores on geometric, topological and transport properties of pore systems for low-permeability porous media. Journal of Hydrology, 575: 327-342. https://dx.doi.org/10.1016/j.jhydrol.2019.05.014

[18] Yang, Y., Zhang, W., Gao, Y., Wan, Y., Su, Y., An, S., Sun, H., Zhang, L., Zhao, J., Liu, L., Liu, P., Liu, Z., Li, A., Yao, J. (2016). Influence of stress sensitivity on microscopic pore structure and fluid flow in porous media. Journal of Natural Gas Science and Engineering, 36: $20-31$. https://dx.doi.org/10.1016/j.jngse.2016.09.061

[19] Zheng, Y., Jeon, B., Xu, D., Wu, Q.M., Zhang, H. (2015). Image segmentation by generalized hierarchical fuzzy Cmeans algorithm. Journal of Intelligent \& Fuzzy Systems, 28(2): 961-973. https://dx.doi.org/10.3233/IFS-141378

[20] Bear, J. (2013). Dynamics of fluids in porous media. Courier Corporation, New York.

[21] Sun, W., Hou, K., Yang, Z., Wen, Y. (2017). X-ray CT three-dimensional reconstruction and discrete element analysis of the cement paste backfill pore structure under uniaxial compression. Construction and Building Materials, 138: 69-78. https://dx.doi.org/10.1016/j.conbuildmat.2017.01.088

[22] Borges, J.A., Pires, L.F., Cássaro, F.A., Roque, W.L., Heck, R.J., Rosa, J.A., Wolf, F.G. (2018). X-ray microtomography analysis of representative elementary volume (REV) of soil morphological and geometrical properties. Soil and Tillage Research, 182: 112-122. https://dx.doi.org/10.1016/j.still.2018.05.004

[23] Gonzalez, J.L., de Faria, E.L., Albuquerque, M.P., Bom, C.R., Freitas, J.C.C., Cremasco, C.W., Correia, M.D. (2018). Representative elementary volume for NMR simulations based on X-ray microtomography of sedimentary rock. Journal of Petroleum Science and Engineering, 166: 906-912. https://dx.doi.org/10.1016/j.petrol.2018.03.104 